\title{
Autophagy promotes invadopodia formation in human ovarian cancer cells via the p62-extracellular signal-regulated kinase $1 / 2$ pathway
}

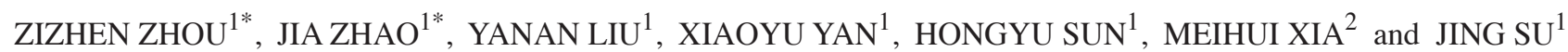 \\ ${ }^{1}$ Key Laboratory of Pathobiology, Ministry of Education, Department of Pathophysiology, \\ Basic College of Medicine, Jilin University; ${ }^{2}$ Department of Obstetrics and Gynecology, \\ The First Hospital, Jilin University, Changchun, Jilin 130021, P.R. China
}

Received December 26, 2019; Accepted May 21, 2021

DOI: $10.3892 / \mathrm{etm} .2021 .10384$

\begin{abstract}
Invasiveness and metastatic potential are among the most essential characteristics of malignant tumors. Furthermore, it has been reported that autophagy and invasion are enhanced when tumor cells are grown in adverse conditions, such as nutritional deficiency and starvation. However, the association between autophagy and invasion remains largely unclear. In the present study, Earle's balanced salt solution (EBSS) was used to induce autophagy and an autophagy inhibitor was used to block autophagy. The results of Transwell assays revealed that autophagy inhibition limited the invasiveness of human ovarian cancer cells. Furthermore, the results of invadopodia formation assay indicated that autophagy stimulated invadopodia formation, and the selective autophagy receptor and signaling adaptor, sequestosome-1 (SQSTM1/p62 or simply p62), was closely associated with invadopodia formation in human ovarian cancer SKOV3 cells. The results of western blot analysis indicated that autophagy induced changes in p62 protein levels and p62 then functioned as a negative regulator of extracellular signal-regulated kinase 1/2 (ERK1/2) activity and invadopodia formation. The interaction between autophagy and invasion may thus be a self-protective mechanism for tumor cells in an unfavorable environment of nutritional deficiency, that maintains their
\end{abstract}

Correspondence to: Dr Meihui Xia, Department of Obstetrics and Gynecology, The First Hospital, Jilin University, 1 Xinmin Street, Changchun, Jilin 130021, P.R. China

E-mail:milansummer@yeah.net

Professor Jing Su, Key Laboratory of Pathobiology, Ministry of Education, Department of Pathophysiology, Basic College of Medicine, Jilin University, 126 Xinmin Street, Changchun, Jilin 130021, P.R. China

E-mail: sujing@jlu.edu.cn

"Contributed equally

Key words: autophagy, invadopodia, p62, ERK1/2 survival and leads to increased invasiveness. An exploration of the intrinsic link between autophagy and invasion may provide a novel theoretical basis to reverse the resistance of tumor cells to a nutritional deficient environment.

\section{Introduction}

Invasion and metastasis are major characteristics of malignant tumors and are the main causes of high mortality in ovarian cancer. During tumor growth, tumor cells are often exposed to nutrient deficiency due to insufficient angiogenesis or drug interventions; however, according to several experimental and clinical research findings, environment deficiency in nutrients does not reduce tumor growth completely. On the contrary, tumor cells invade the matrix and blood vessels in order to survive and subsequently metastasize to other organs and tissues where nutrition is higher, causing high mortality (1). Starvation and nutrient deficiency have also been reported to induce autophagy in tumor cells through the decomposition of their organelles and other organic substrates. This provides raw materials in the form of amino acids and other necessary small molecules that maintain cell survival (2). These findings suggest that autophagy and invasion constitute a self-protective mechanism of tumor cells under nutrient deficient conditions that acts as a bottleneck, impeding anti-angiogenesis drug effectiveness (3-5). Therefore, an exploration of the intrinsic link between autophagy and invasion could reveal the tumor cell metastasis mechanism and provide a novel theoretical basis to specifically target metastasis and reverse tumor cell resistance in nutrient deficiency conditions.

Invadopodia are actin-rich protrusions that localize proteolytic activity to areas of the cell in contact with the extracellular matrix (ECM). The ability to form invadopodia is closely associated with the invasive and metastatic potential of a tumor. A wide variety of actin-interacting and scaffolding proteins are involved in invadopodia formation, including cortactin, tyrosine kinase substrate with five Src homology SH3 domains, fascin, neural Wiskott-Aldrich syndrome protein (N-WASP), and actin-related protein complex 2/3 (Arp2/3 complex) $(6,7)$. In particular, the actin-bundling protein, cortactin, appears to play an integral role in invadopodia formation (6-9). Src kinase 
activity and cortactin phosphorylation are absolute requirements for invadopodia formation, through the phosphorylation of serine residues 405 and 418 within the PR domain of cortactin by extracellular signal-regulated kinase (ERK1/2) or p21-activated kinase 1, which according to previous studies enhances cortactin SH3 domain binding to N-WASP (10). As a result, it has been suggested that S405/S418 phosphorylation plays a critical role in the regulation of the cellular actin polymerization, which is necessary for the promotion of invadopodia function $(11,12)$.

The selective autophagy receptor and signaling adaptor, sequestosome-1 (SQSTM1/p62, hereafter referred to as p62) is a multidomain protein. The presence of an LC3-interacting region enables p62 to bind to the autophagy protein microtubule-associated protein 1A/1B light chain 3B (LC3), and the ubiquitin-associated domain binds to ubiquitin to mediate the selective degradation of ubiquitinated cargo by autophagy. p62 contains a protein-protein interaction module (PB1) that facilitates its oligomerization and is rich in protein-interacting sequences, that function as a signaling hub through its interaction with key components of signaling mechanisms including the Ras/Raf/mitogen-activated protein kinase pathway and nuclear factor $\kappa$ B pathway (13-17).

Tumors often exist in low nutrition environments during their growth or treatment; however, may overcome this by invading other organs and subsequently causing high mortality.

\section{Materials and methods}

Reagents and antibodies. ViaFect Transfection Reagent was purchased from Promega Corporation. 3-(4,5-Dimethylthiazol2-yl)-2,5-diphenyltetrazolium bromide(MTT), Hoechst 33342, Gelatin from pig skin, and fetal bovine serum (FBS) were purchased from Sigma-Aldrich; Merck KGaA. The nuclear and cytoplasmic protein extraction kit was purchased from Beyotime Institute of Biotechnology. The ERK1/2 inhibitor, SCH772984, chloroquine (CQ) and 3-methyladenine (3-MA) were purchased from MedChemExpress. The following antibodies were used: Anti-p62 (cat. no. ab91526; Abcam), anti-cortactin (cat. no. ab269977; Abcam); anti- $\beta$-actin (cat. no. MA5-15452; ProteinTech Group, Inc.); anti-ERK1/2 (cat. no. sc-514302; Santa Cruz Biotechnology, Inc.) and anti-LC3 (cat. no. sc-398822; Santa Cruz Biotechnology, Inc.).

Cell lines and cell culture. Human ovarian carcinoma cells (SKOV3 cells; cat. no. TCHu185) were purchased from the The Cell Bank of Type Culture Collection of the Chinese Academy of Sciences and Peking Union Medical College. SKOV3 cells were cultured in Roswell Park Memorial Institute (RPMI)1640 culture medium (Gibco; Thermo Fisher Scientific, Inc.) supplemented with $10 \%$ fetal bovine serum (Invitrogen; Thermo Fisher Scientific, Inc.) at $37^{\circ} \mathrm{C}$ in $5 \% \mathrm{CO}_{2}$.

Autophagy evaluation. Autophagy promoted by amino acid starvation was examined, with the use of $70 \%$ confluent cells, washed 3 times with modified Earle's balanced salt solution (EBSS; $0.265 \mathrm{~g} / 1 \mathrm{CaCl}_{2} . \mathrm{H}_{2} \mathrm{O}, 0.09767 \mathrm{~g} / 1 \mathrm{MgSO} 4,0.4 \mathrm{~g} / 1$ $\mathrm{KCl}, 6.8 \mathrm{~g} / 1 \mathrm{NaCl}, 0.122 \mathrm{~g} / 1 \mathrm{NaH}_{2} \mathrm{PO}_{4}, 1.0 \mathrm{~g} / 1 \mathrm{D}-G$-Gucose, 0.011 g/l Phenol Red.Na, 2.2 g/l NaHCO 3 ; Sigma-Aldrich; Merck $\mathrm{KGaA}$ ) and then incubated with EBSS at $37^{\circ} \mathrm{C}$ in $5 \%$
$\mathrm{CO}_{2}$. Autophagy evaluation was performed in accordance with previously published studies (18-21).

Transwell invasion assay. Cells were serum-starved for $24 \mathrm{~h}$, harvested and resuspended in medium containing 1\% bovine serum albumin or EBSS. For the invasion assay, the top chambers were coated with Matrigel (cat. no. 354234; Corning, Inc.). Cells $\left(5 \times 10^{4}\right)$ were added to the top chambers of 24-well Transwell plates (Costar; Corning, Inc.). Medium containing $10 \%$ fetal bovine serum was added to the bottom chambers. Cells were incubated at $37^{\circ} \mathrm{C}$ for $0-24 \mathrm{~h}$. The cells were fixed in $0.1 \%$ glutaraldehyde-PBS for $20 \mathrm{~min}$ and stained with $0.2 \%$ crystal violet (Sigma-Aldrich; Merck KGaA) for $1 \mathrm{~h}$ at room temperature. Non-motile cells on the top of each filter were then removed using a cotton swab. The number of invasive cells was counted using an Olympus IX-71 inverted microscope (Olympus Corporation).

Invadopodia formation assay. Cells were plated on gelatin matrix in RPMI-1640 medium or EBSS. At the indicated time points $(0,2,4,8,16$ and $24 \mathrm{~h})$, the cells were washed, fixed and permeabilized with $4 \%$ paraformaldehyde (cat. no. 158127; Sigma-Aldrich; Merck KGaA) and 0.2\% Triton-X (cat. no. A93443; Sigma-Aldrich; Merck KGa) for $20 \mathrm{~min}$ at $37^{\circ} \mathrm{C}$. Additionally, the cells were stained to view punctuate structures where F-actin and cortactin colocalized. Cells were incubated for $37^{\circ} \mathrm{C}, 1 \mathrm{~h}$ with anti-cortactin (1:100; cat. no. ab269977; Abcam) antibody and TRITC-phalloidin (1:1,000; cat. no. 41-6559-05; Invitrogen; Thermo Fisher Scientific, Inc.). Images were acquired by an Olympus FV1000 confocal laser microscope (Olympus Corporation) and measured using ImageJ software 1.8.0 (National Institutes of Health).

Cell transfection. p62-siRNA and non-target siRNA (Scramble) were obtained from Shanghai GeneChem Co., Ltd. The p62-siRNA (si-p62) sequence was GAC-ATC-TTCCGAATC-TAC-A and the non-target siRNA (Scramble) was TTC-TCC-GAA-CGT-GTC-ACG-T. The pcDNA3.1-p62 and empty pcDNA3.1 vector (NC) were constructed by Sangon Biotech Co., Ltd. Cell transfection was performed as previously described (22). A total of $25 \times 10^{4}$ cells were plated in 6 -well plates. After $36 \mathrm{~h}$ culture, cells were transfected with siRNA (2 $\mu \mathrm{g} /$ well) or plasmids $(2.5 \mu \mathrm{g} /$ well $)$ using Lipofectamine $^{\mathrm{TM}} 2000$ Transfection Reagent (Invitrogen; Thermo Fisher Scientific, Inc.11668019) according to the manufacturer's instructions. Cells were incubated for 1-3 days at $37^{\circ} \mathrm{C}$ and then used in subsequent experiments.

Cell viability assays. A total of $8 \times 10^{3}$ cells/well were seeded in 96-well plates. Cells were cultured for $24 \mathrm{~h}$, and then the cells were treated with EBSS or various concentrations of CQ $(0,10$, $25,50$ or $100 \mu \mathrm{M}) / 3$-MA $(0,1,2,4,8$ or $10 \mathrm{mM})$. MTT assay was used to evaluate cell viability. MTT $(5 \mathrm{mg} / \mathrm{ml})$ was added to the cells and DMSO was used to dissolve the formazan. The absorbance was measured at $570 \mathrm{~nm}$ using a Vmax Microplate Reader (Molecular Devices, LLC).

Western blot analysis. Protein expression was examined by with western blot analysis, as previously described (22). 
A

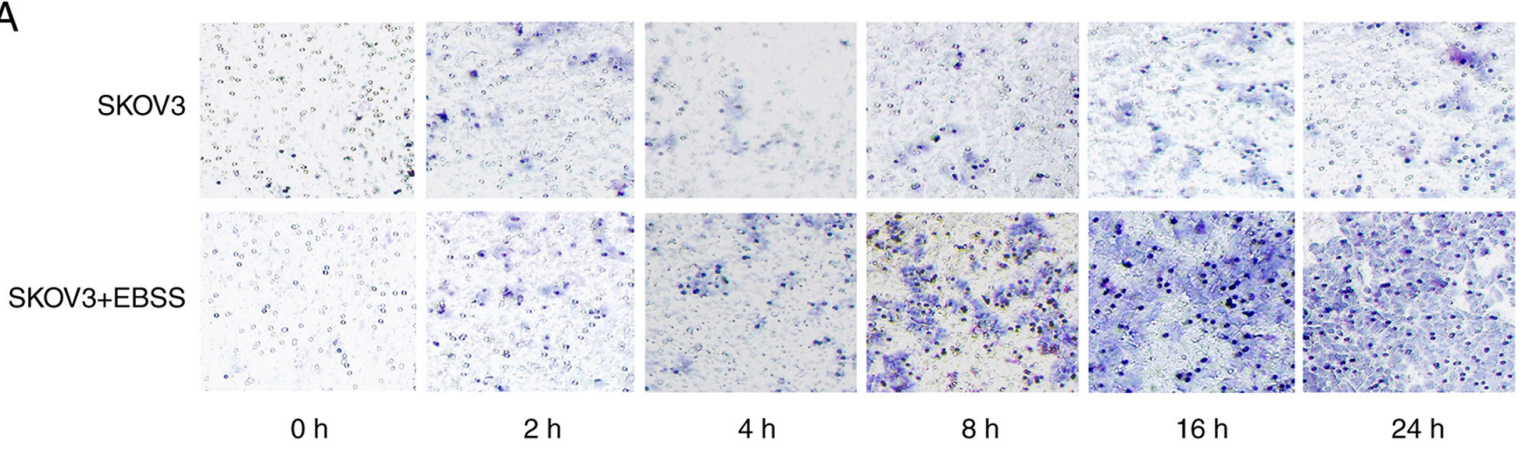

B

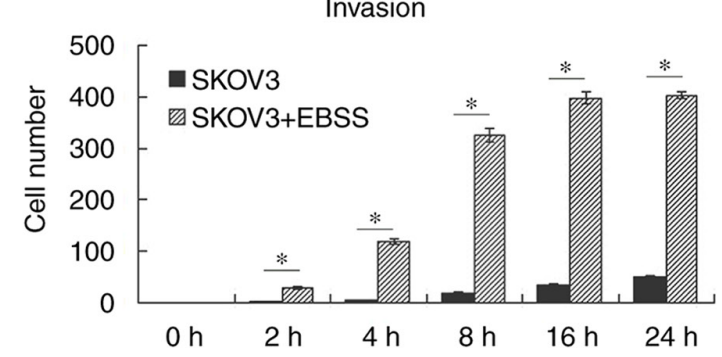

D

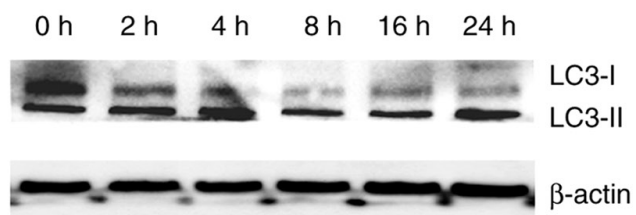

C

Cell viability

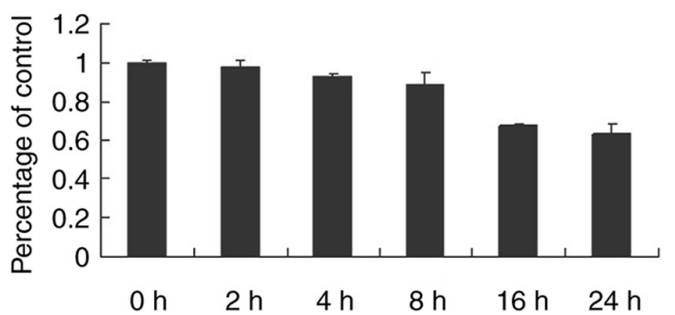

E

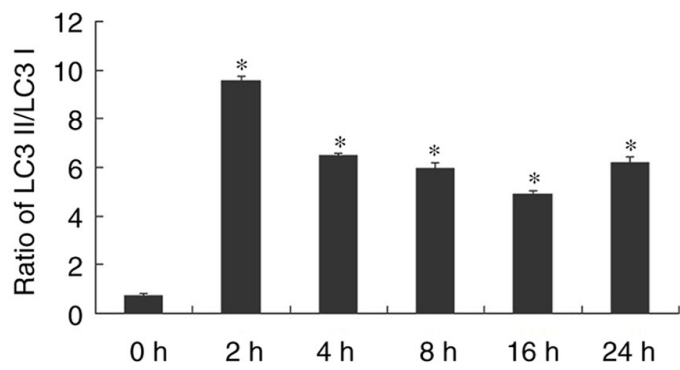

Figure 1. EBSS enhances the invasion and autophagy of human ovarian cancer cells. (A) Representative images of Transwell analysis (magnification, x200). (B) Invasiveness of human ovarian cancer cell SKOV3 treated with EBSS was detected by Transwell analysis. (C) Cell viability of EBSS-treated SKOV3 cells was detected by MTT assay. (D) LC3 protein expression in EBSS-treated SKOV3 cells was detected by western blot analysis. (E) Densitometric analysis of LC3 expression levels ("P<0.05, compared with control). EBSS, Earle's balanced salt solution; LC3, Microtubule-associated protein 1A/1B light chain 3B.

Protein was extracted using M-PER ${ }^{\mathrm{TM}}$ Mammalian Protein Extraction Reagent (cat. no. 78505; Thermo Fisher Scientific), the protein concentration was detected by bicinchoninic acid method. Protein was loaded (40 $\mu \mathrm{g}$ per lane) into $10 \%$ gel and proteins were transferred to PVDF membrane. Membrane was blocked in $1 \%$ BSA at room temperature for $1 \mathrm{~h}$, and incubated with the following primary antibodies overnight at $4^{\circ} \mathrm{C}$ : Anti-p62 (1:1,000; cat. no. ab91526; Abcam), anti-cortactin (1:1,000; cat. no. ab269977; Abcam), anti-p-cortactin (1:1,000; cat. no. ab47768; Abcam), anti$\beta$-actin (1:1,000; cat. no. MA5-15452; ProteinTech Group, Inc.), anti-ERK1/2 (1:1,000; cat. no. sc-514302; Santa Cruz Biotechnology, Inc.), anti-p-ERK1/2 (1:500; cat. no. sc-136521; Santa Cruz Biotechnology, Inc.) and anti-LC3 (1:1,000; cat. no. sc-398822; Santa Cruz Biotechnology, Inc.) using antibody diluents (cat. no. U3635; Sigma-Aldrich; Merck KGaA). Goat anti-mouse horseradish peroxidaseconjugated secondary antibodies were incubated $(1: 200$; cat. no. SA00001; ProteinTech Group, Inc.) according to the manufacturer's instructions. Then, immunodetection was performed using ECL reagent (Thermo Fisher Scientific,
Inc.) and visualized using a Syngene Bio Imaging System (Syngene Europe), and densitometry was performed using ImageJ software v1.8.0 (National Institutes of Health).

Immunofluorescence staining. SKOV3 cells were cultured on coverslips at a density of $5 \times 10^{4}$ cells/well in $500 \mu 1$ of complete medium. Following treatment, the SKOV3 cells were washed with cold PBS three times and fixed in $4 \%$ (w/v) paraformaldehyde/PBS for $20 \mathrm{~min}$ and then washed with cold PBS three times. The fixed cells were subsequently digested by protein enzyme $\mathrm{K}$ for $1 \mathrm{~min}$ and washed with PBS twice. The cells were then incubated with $0.1 \%$ (v/v) Triton X-100 for 6-10 min, washed once with PBS, and then blocked for $30 \mathrm{~min}$ in $5 \%(\mathrm{v} / \mathrm{v})$ non-immune animal serum/PBS. The cells were incubated with the anti-cortactin primary antibody (1:100; cat. no. ab269977; Abcam) overnight and washed three times with PBS. They were then incubated with goat anti-mouse IgG secondary antibody conjugated to Alexa Fluor ${ }^{\circledR}$ Plus 488 (1:400; cat. no. A32723; Thermo Fisher Scientific) for $30 \mathrm{~min}$ in the dark. Plates were washed three times in PBS, treated with Hoechst $33342 / \mathrm{H}_{2} \mathrm{O}$ 
A

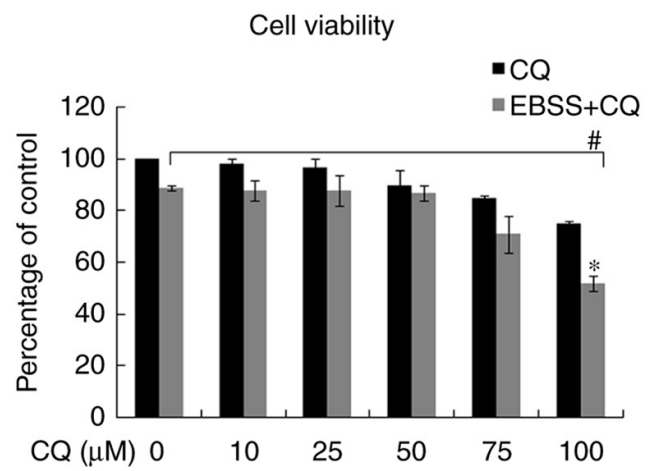

C

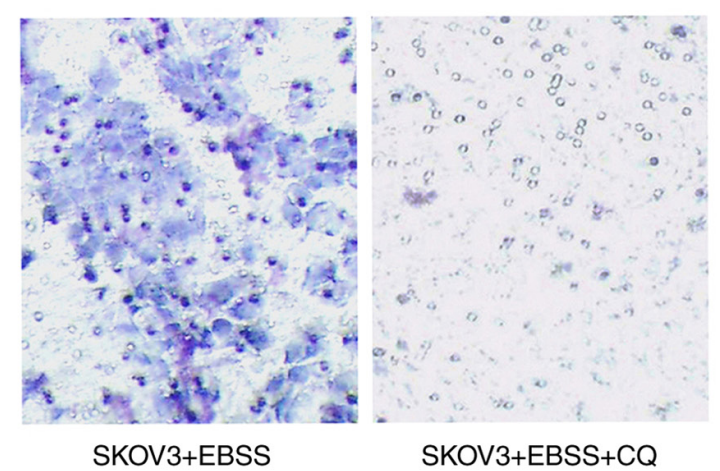

E

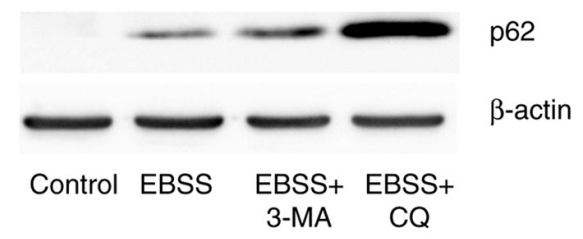

B

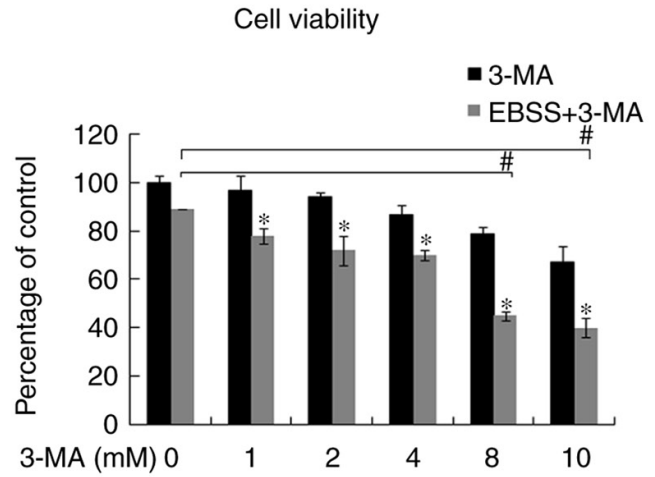

D

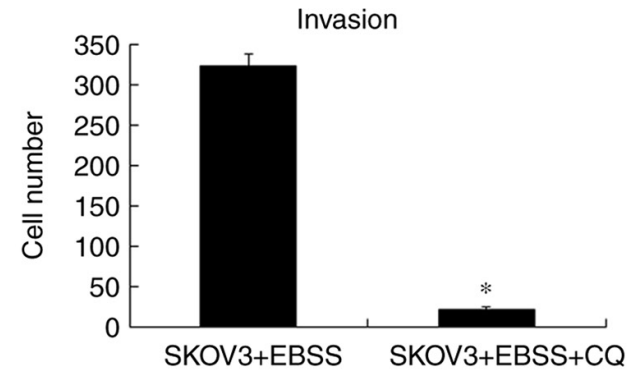

F

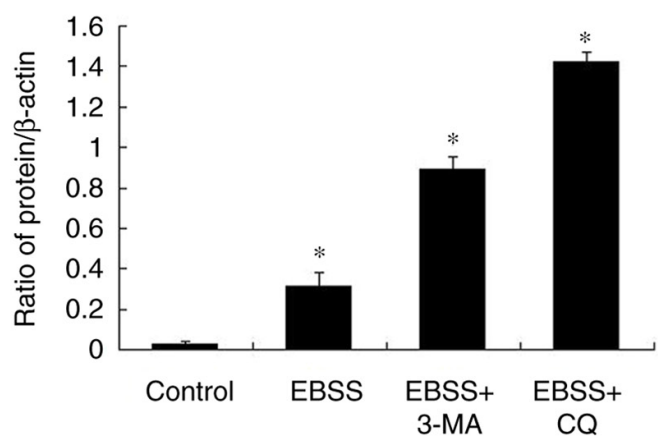

Figure 2. Autophagy inhibition reduces invasion of human ovarian cancer cells. (A) Cell viability of SKOV3 (with or without EBSS) treated with CQ was detected by MTT analysis ( ${ }^{*} \mathrm{P}<0.05$ compared with CQ at the same concentration; ${ }^{*} \mathrm{P}<0.05$ compared with EBSS). (B) Viability of SKOV3 cells (with or without EBSS) treated with 3-MA was detected by MTT assay ( ${ }^{*} \mathrm{P}<0.05$ compared with 3-MA at the same concentration; ${ }^{\#} \mathrm{P}<0.05$ compared with EBSS). (C) Representative images of Transwell assay (magnification, $\mathrm{x} 200$ ). (D) Invasion of SKOV3 human ovarian cancer cells treated with EBSS and CQ (50 $\mu \mathrm{M}$ ) detected by Transwell assay ( ${ }^{*} \mathrm{P}<0.05$ compared with control). (E) p62 protein level in SKOV3 human ovarian cancer cells treated with EBSS and CQ $(50 \mu \mathrm{M}) / 3$-MA ( $4 \mathrm{mM})$ detected by western blot analysis. (F) Densitometric analysis of p62 expression levels (*P<0.05 compared with control). EBSS, Earle's balanced salt solution; p62, selective autophagy receptor and signaling adaptor sequestosome-1; CQ, chloroquine; 3-MA, 3-methyladenine.

(1 $\mu \mathrm{g} / \mathrm{ml})$ for $2 \mathrm{~min}$, and then washed three times with PBS. The cells were examined using an Olympus FV1000 confocal laser microscope (Olympus Corporation; magnification, $\mathrm{x} 400)$.

Statistical analysis. Statistical analysis was performed using the Statistical Package for the Social Sciences, version 22 (IBM Corp.). All data are presented as the mean \pm SD. The unpaired Student's t-test was applied, in order to perform comparisons between two groups. One-way ANOVA followed by Tukey's post hoc test was used to analyze datasets with more than two groups. Two-way ANOVA followed by Bonferroni's post hoc test was performed to evaluate the effects of concentration and EBSS. $\mathrm{P}<0.05$ was considered to indicate a statistically significant difference.

\section{Results}

Autophagy inhibition reduces the invasion of human ovarian cancer cells. The ability of cancer cells to metastasize is dependent on their capability to invade surrounding tissues and migrate from the primary tumor site. In the present study, it was demonstrated that EBSS increased the invasion of the human ovarian cancer cell line, SKOV3. EBSS is a balanced salt solution and does not contain amino acids or other active substances, thus providing a nutrient deficient environment. SKOV3 cells cultured in EBSS exhibited an increased invasion compared with the control cells (Fig. 1A and B). Fig. 1C further confirmed that the increased invasion was not due to cell viability increase. As EBSS has been previously reported to induce autophagy (18-21), in the 
A
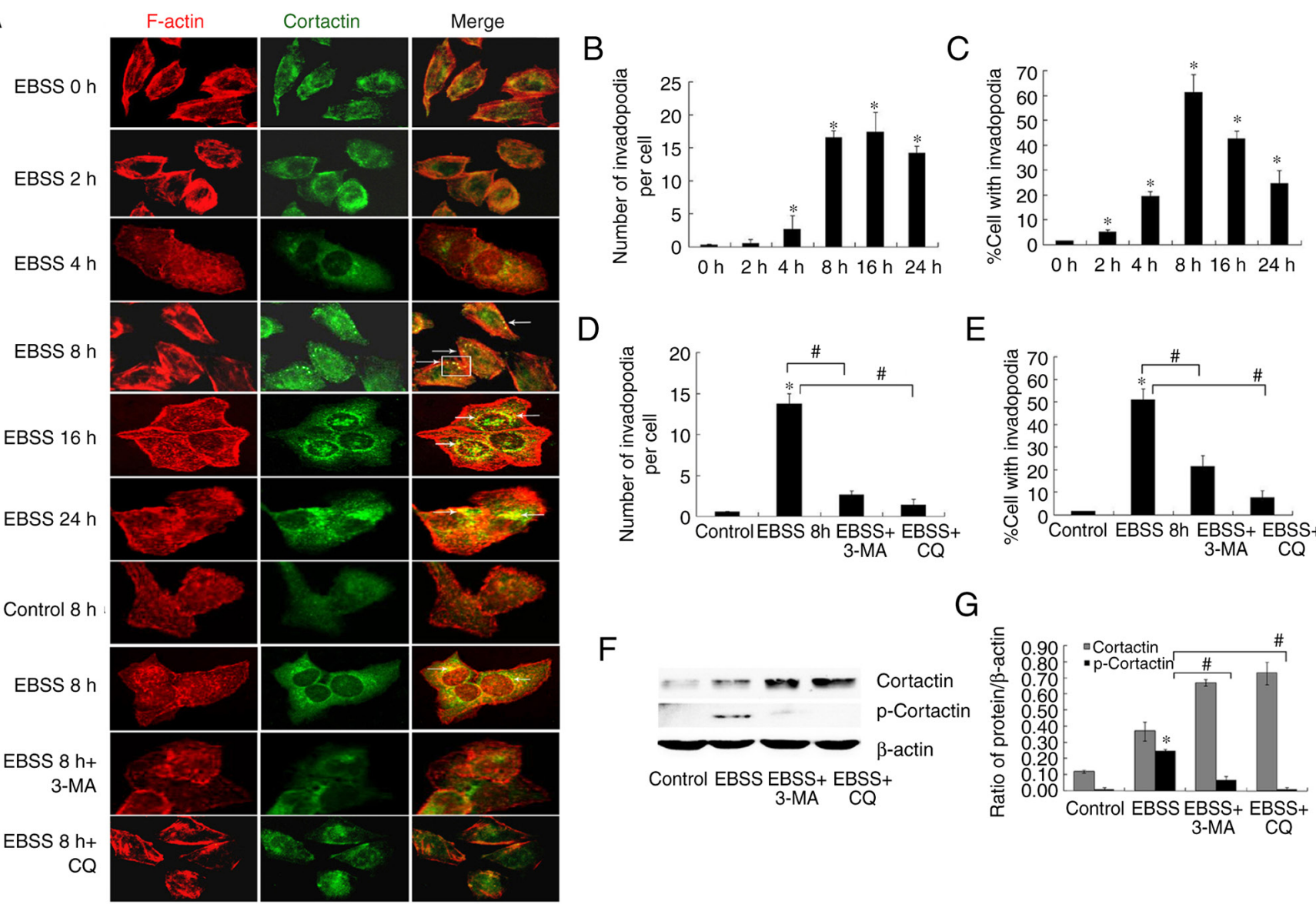

E

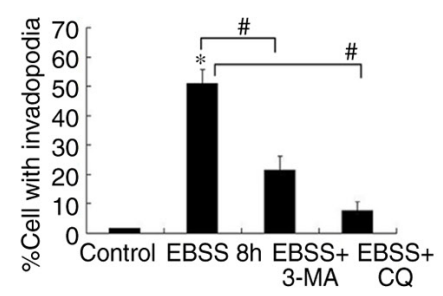

G

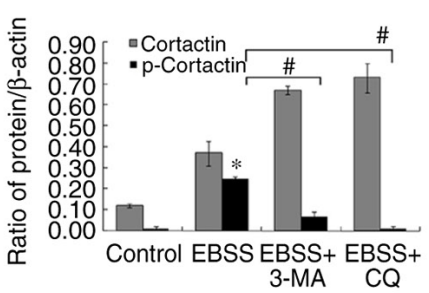

Figure 3. Autophagy stimulates invadopodia formation. Invadopodia formation of EBSS treated SKOV3 cells was examined by immunofluorescence. Cells were plated on gelatin matrix in RPMI-1640 medium or EBSS. Cells were incubated for $1 \mathrm{~h}$ with anti-cortactin antibody and TRITC-phalloidin. Images were acquired using an Olympus FV1000 confocal laser microscope and measured by ImageJ software. (A) Representative pictures of invadopodia formation (magnification, x400). (B and C) Data analysis of invadopodia formation of EBSS treated SKOV3 cells (" $\mathrm{P}<0.05$ compared with control). (D and E) Data analysis of invadopodia formation of autophagy inhibitor-treated SKOV3 cells. ("P $<0.05$ compared with control, ${ }^{*} \mathrm{P}<0.05$ compared with EBSS $\left.8 \mathrm{~h}\right)$. (F) Protein and phosphorylation levels of cortactin were detected by western blot analysis. (G) Densitometric analysis of protein and phosphorylation levels of cortactin $\left(\right.$ $\mathrm{P}<0.05$ compared with control; ${ }^{*} \mathrm{P}<0.05$ compared with EBSS). EBSS, Earle's balanced salt solution.

present study, western blot analysis performed to examine autophagy in SKOV3 cells treated with EBSS through the detection of LC3 protein expression. LC3II protein levels were significantly increased following the treatment of SKOV 3 cells with EBSS for $2 \mathrm{~h}$, peaked after $8 \mathrm{~h}$ of treatment and then decreased (Fig. 1D and E). Subsequently, in order to examine the effects of autophagy in the regulation of cell migration and invasion, the early autophagy inhibitor, 3-MA, and the late autophagy inhibitor, CQ, were used in order to block autophagy. The results of cell viability assay revealed that there was no significant difference in cell viability between EBSS and EBSS + CQ $(50 \mu \mathrm{M}) /$ EBSS and EBSS +3-MA (4 mM) (Fig. 2A and B); the appropriate concentration of CQ and 3-MA (50 $\mu \mathrm{M}$ and $4 \mathrm{mM}$, respectively) was then selected to perform the following assays. According to the results of western blot analysis, autophagy was blocked by both 3 -MA $(4 \mathrm{mM})$ and CQ $(50 \mu \mathrm{M})$, as evidenced by the increased expression of p62 (Fig. 2E and F). Transwell assay for cell invasion detection revealed that EBSS-induced invasion was inhibited when autophagy was blocked by CQ $(50 \mu \mathrm{M})$ (Fig. 2C and D), while there was no significant difference in cell viability between EBSS and EBSS + CQ $(50 \mu \mathrm{M})$ treatment (Fig. 2A). The aforementioned results indicated that autophagy inhibition reduced human ovarian cancer cell invasiveness.

Autophagy stimulates invadopodia formation. In an attempt to fully understand the association between autophagy and invasion, the formation of invadopodia, which are critical structures associated with tumor invasion, was examined. Invadopodia formation is a dynamic process, characterized by the co-localization of cortactin and F-actin in punctate or rosette structures $(6,7)$. The co-localization of F-actin and cortactin as compared with control cells was observed (Fig. 3A). The application of EBSS treatment for $8 \mathrm{~h}$ stimulated invadopodia formation more robustly than a shorter 2-h treatment. The use of an autophagy inhibitor reduced the number of cells with active invadopodia (Fig. 3A, D and E). Moreover, treatment of the cells with 3-MA or CQ blocked EBSS-induced invadopodia formation, indicating the autophagy implication in the regulation of this process.

Invadopodia formation is dependent on the activity of cortactin, and cortactin phosphorylation is also associated with the increased activity and maturation of invadopodia $(8,9)$. In the present study, it was observed that the stimulation of SKOV3 cells with EBSS increased cortactin phosphorylation. 
A

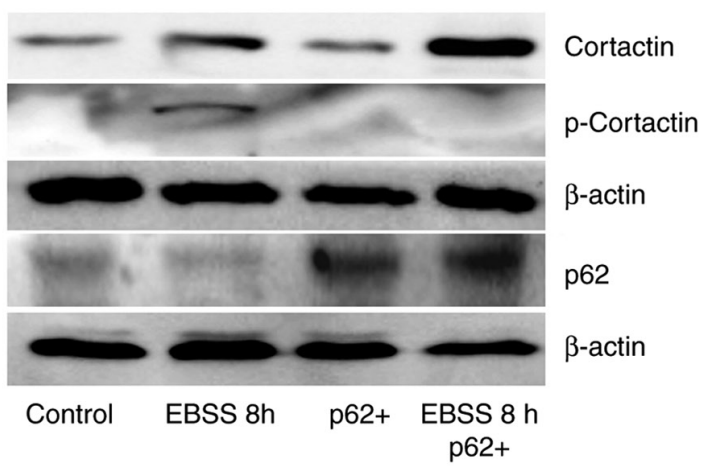

C

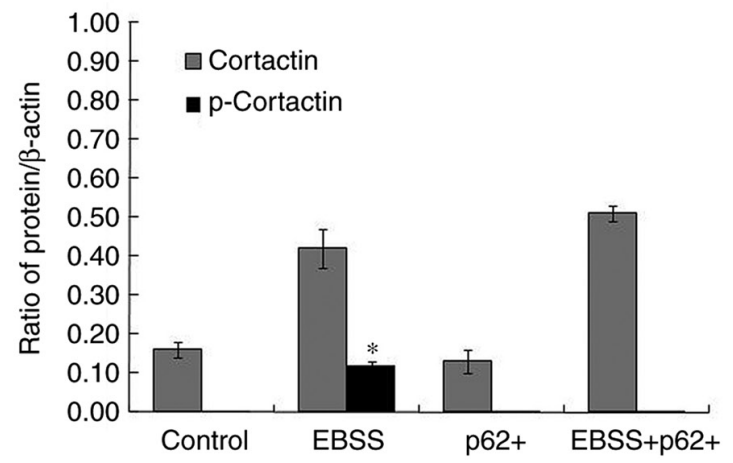

E

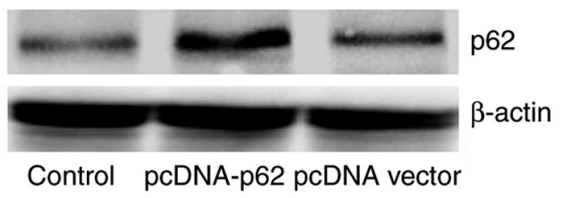

G

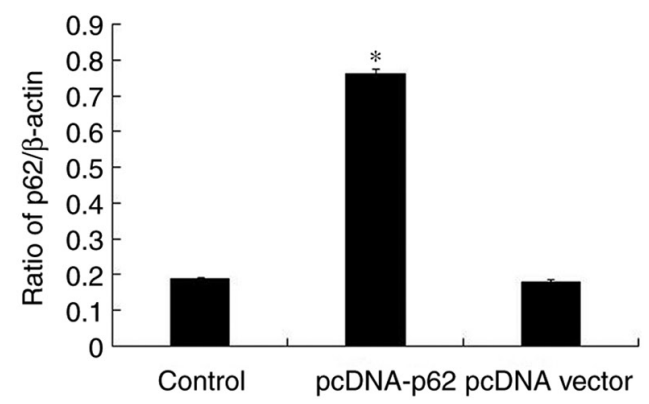

B

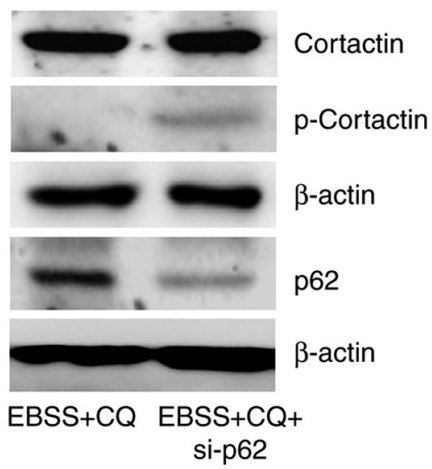

D

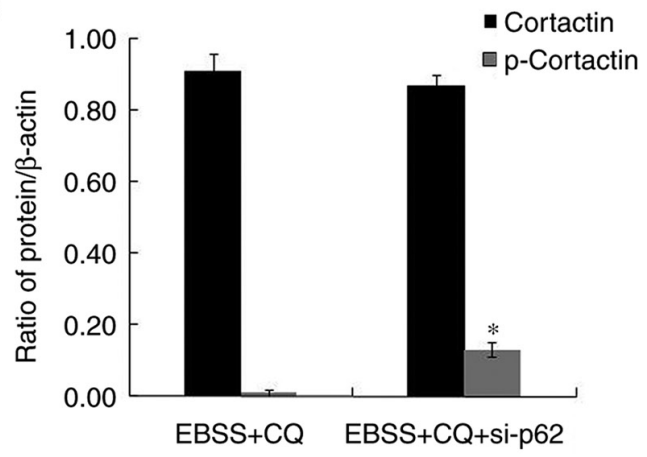

F

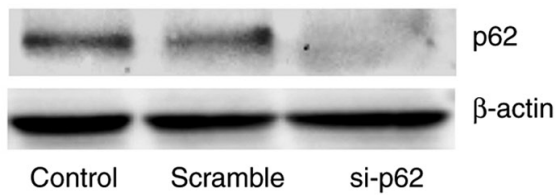

$\mathrm{H}$

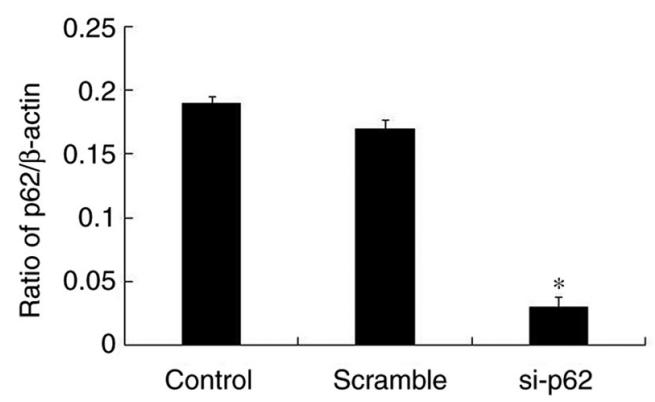

Figure 4. Effect of p62 on cortactin expression and phosphorylation. (A and B) Protein and phosphorylation levels of cortactin were detected by western blot analysis. (C and D) Densitometric analysis of protein and phosphorylation levels of cortactin. (E and F) Protein levels of p62 were detected by western blot analysis. ( $\mathrm{G}$ and $\mathrm{H}$ ) Densitometric analysis of protein levels of p62 ("P $<0.05$ compared with control). EBSS, Earle's balanced salt solution; p62, selective autophagy receptor and signaling adaptor sequestosome-1.

By contrast, although the protein level of cortactin increased when autophagy was blocked, cortactin phosphorylation was inhibited (Fig. 3F and G).

p62 is closely associated with invadopodia formation in human ovarian cancer SKOV3 cells. p62 undergoes dynamic changes that accompany different autophagy conditions and interacts with key components of various signaling mechanisms. As a consequence, the present study then explored the role of p62 in invadopodia formation. As shown in Fig. 2E, p62 degradation was observed along with autophagy following EBSS treatment for $8 \mathrm{~h}$; conversely, p62 accumulated when autophagy was blocked. It was also demonstrated that cortactin phosphorylation was reduced in p62-overexpressing cells, following $8 \mathrm{~h}$ of EBSS treatment (Fig. 4A and C), while the depletion of p62 in autophagy-blocked cells partially recovered cortactin phosphorylation (Fig. 4B and D). Fig. 4E-H showed that the transfections were successful. Although the presence or absence of EBSS resulted in unequal cortactin protein levels, these results revealed that the increased expression of cortactin was the stress response of cells to the starvation environment, while p62 regulated cortactin phosphorylation. Moreover, it 
A

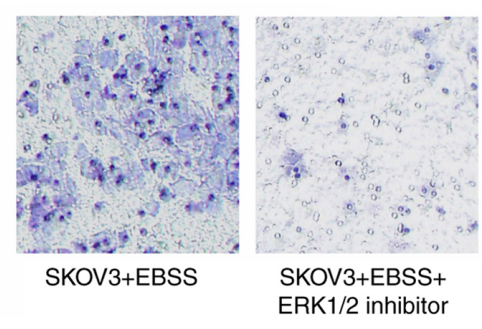

C
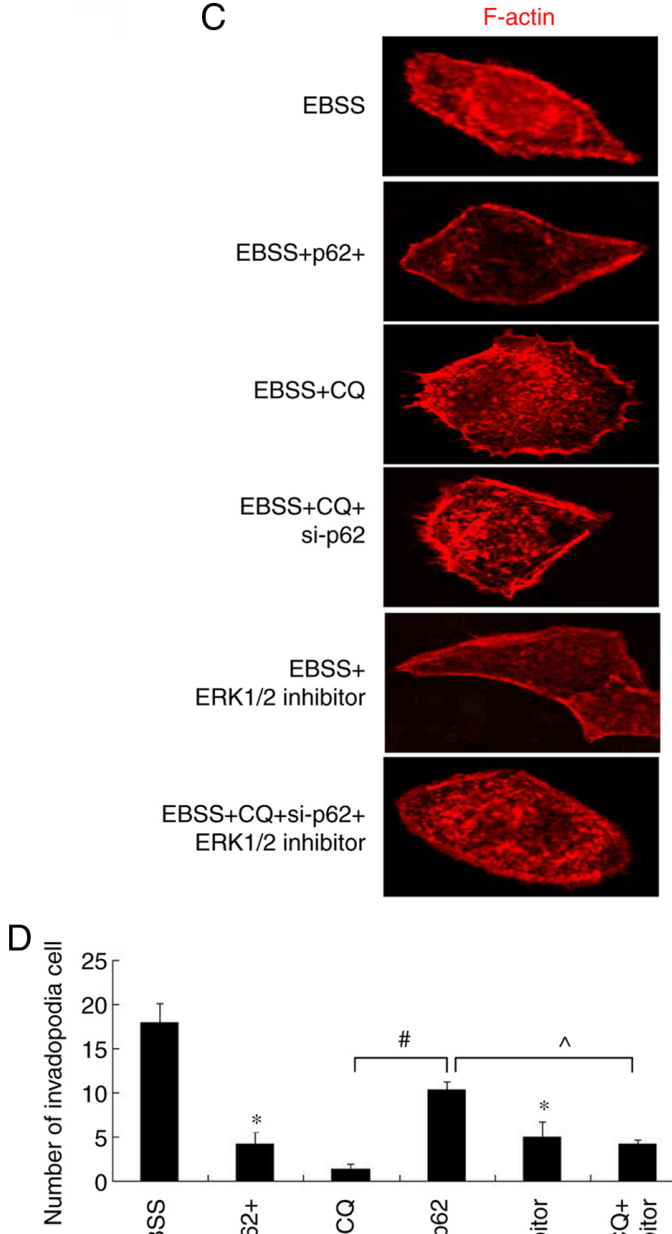

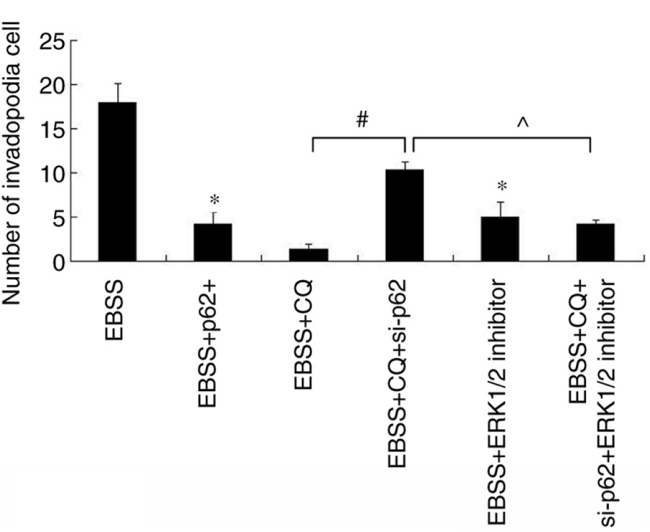

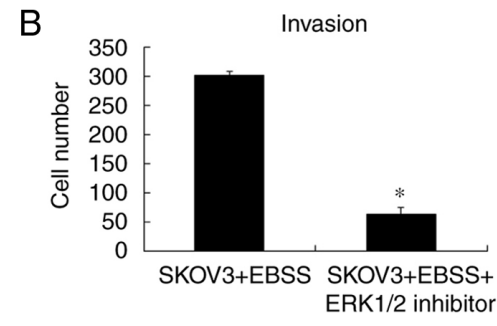
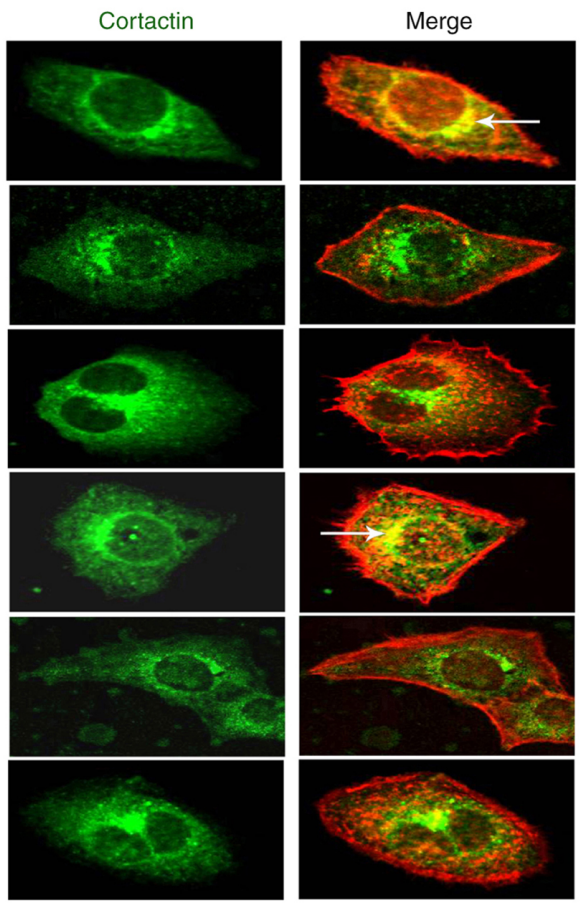

$\mathrm{E}$

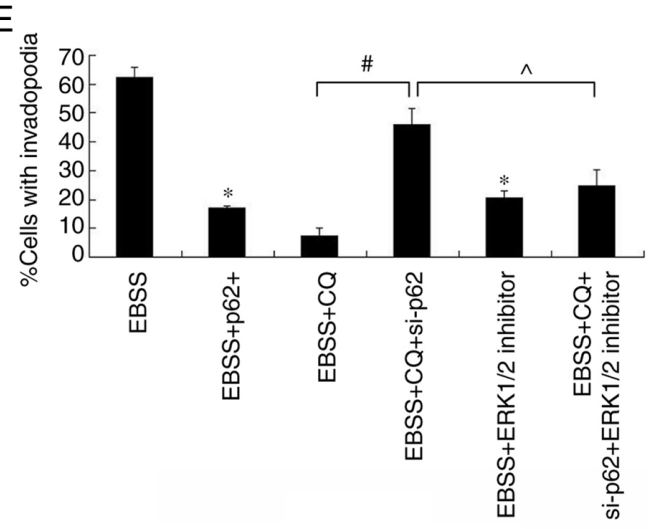

Figure 5. Effect of p62 on invadopodia formation of human ovarian cancer SKOV3 cells. Invadopodia formation was examined by immunofluorescence. (A) Representative images of invadopodia formation (magnification, $\mathrm{x} 400$ ). (B and C) Data analysis of invadopodia formation of differentially treated SKOV3 cells ("P<0.05 compared with EBSS; " $\mathrm{P}<0.05$ compared with EBSS + CQ; ${ }^{\wedge} \mathrm{P}<0.05$ compared with EBSS + CQ + si-p62). (D) Representative images of Transwell assay (magnification, x200). (E) Invasion of human ovarian cancer cell SKOV3 treated with EBSS and ERK1/2 inhibitor was detected by Transwell assay ('P<0.05 compared with control). EBSS, Earle's balanced salt solution; $\mathrm{CQ}$, chloroquine.

was observed that exogenous p62 inhibited autophagy-induced invadopodia formation, while p62 depletion in autophagyblocked cells partially recovered the ability of invadopodia formation (Fig. 5A-C). Taken together, these results suggest that the p62 quantity is key to invadopodia formation regulation in autophagy.

p62 regulates ERK1/2 to function in autophagy-induced invadopodia formation. Previous studies have demonstrated that ERK1/2 promotes cortactin phosphorylation $(9,10)$, and that the p62 PB1 domain interacts with ERK1/2, in order to inhibit its activity (23). To further investigate the mechanisms of p62 in the regulation of invadopodia formation, the effect of an ERK1/2 inhibitor on cell invasion and invadopodia formation was examined. As shown in Fig. 5, the ERK1/2 inhibitor reduced autophagy-induced cell invasion (Fig. 5A and B) and invadopodia formation (Fig. 5C-E), indicating that ERK1/2 is essential for this process. This reduction in invadopodia 
A

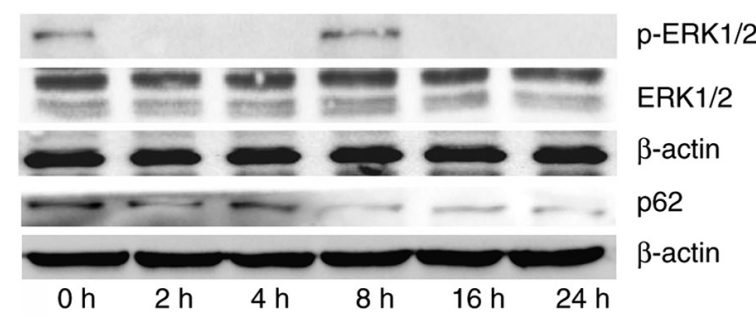

B

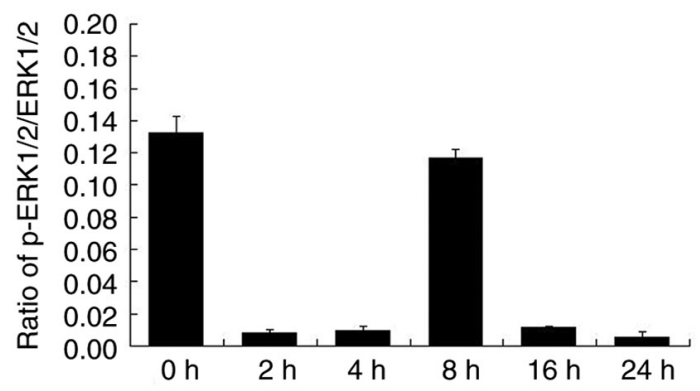

C

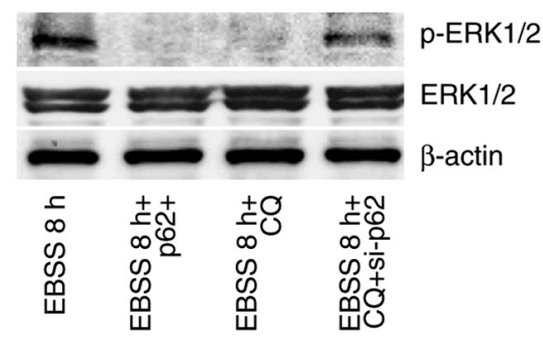

D

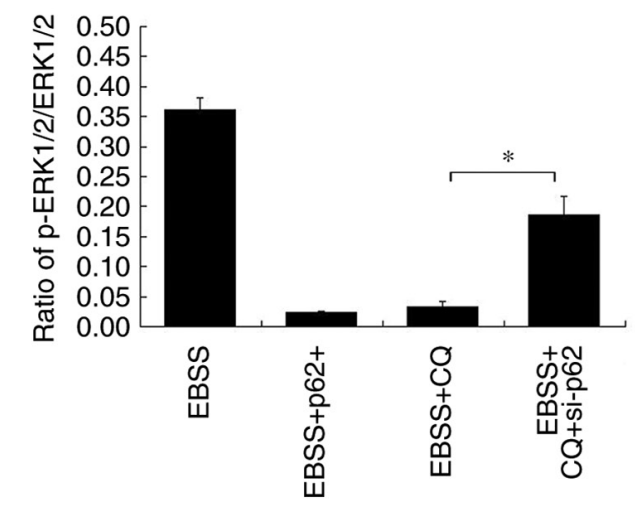

Figure 6. Effect of p62 on ERK1/2 expression and phosphorylation. (A and B) Relative protein expression of ERK1/2 examined by western blot analysis and subsequent densitometric analysis. (C and D) Phosphorylation evaluation of ERK1/2, detected by western blot analysis and subsequent densitometric analysis ( $\mathrm{P}<0.05$ compared with control). EBSS, Earle's balanced salt solution; ERK1/2, extracellular signal-regulated kinases 1/2.

formation was recovered by p62 depletion in autophagyblocked cells (Fig. 5C).

To better understand the mechanisms through which autophagy regulates invadopodia formation, the expression and phosphorylation of ERK1/2 in SKOV3 cells subjected to different treatments were detected. ERK1/2 phosphorylation was shown to vary at different times of EBSS treatment (Fig. 6A and B).

EBSS treatment initially reduced the phosphorylation of ERK1/2, an effect which was reversed in due time. p-ERK1/2 expression was increased after $8 \mathrm{~h}$ of treatment with EBSS (Fig. 6A and B); this finding is consistent with the results shown in Fig. 3, according to which the invadopodia formation detection commenced at $8 \mathrm{~h}$. Subsequently, the p-ERK1/2 levels were observed at different p62 expression levels. In EBSS $8 \mathrm{~h}+$ p62 $2^{+}$cells (cells overexpressing p62 and treated with EBSS), p-ERK1/2 levels decreased. In the EBSS $8 \mathrm{~h}+$ CQ-treated cells, $\mathrm{p}$-ERK1/2 levels decreased. In the EBSS $8 \mathrm{~h}$ $+\mathrm{CQ}+$ si-p62 cells, p-ERK1/2 expression levels increased. (Fig. 6C and D). According to the aforementioned results, ERK1/2 phosphorylation was regulated by p62 in autophagyinduced invadopodia formation.

\section{Discussion}

The present study aimed to elucidate the mechanisms through which autophagy regulates invadopodia formation, implicating p62 and ERK1/2 in this process. Although there is some controversy about the interactions between autophagy and metastasis, to the best of our knowledge, this is the first demonstration of the effect of autophagy on invadopodia.

ECM degradation is considered a key step in promoting tumor invasion and metastasis. Extensive studies have largely focused on secreted matrix metalloproteinases as key proteases in tumor invasion (24-26). More recently, invadopodia have been shown to be critical structures associated with tumor invasion (27) that restrict protease activity to areas of the cell in direct contact with the ECM, thus precisely controlling cell invasion in vivo. In the present study, it was demonstrated that autophagy is both necessary and sufficient to promote invadopodia formation. Furthermore, the existence of important regulatory mechanisms between autophagy and invasion has been elucidated.

The mechanisms through which invadopodia formation is regulated at the molecular level are poorly understood. Cortactin has been reported as a pre-requisite for invadopodia stabilization and maturation. Furthermore, in previous studies, cortactin phosphorylation has been shown to result in actin assembly for the direct formation of actin-rich invadopodia puncta $(8,28)$. ERK1/2, Src and certain other kinases may be implicated in cortactin phosphorylation and the promotion of invadopodia formation $(10,11,29)$. The formation of invadopodia includes structural maturation and functional maturation. In the present study, the structural maturation of invadopodia was depicted by invadopodia formation assay. In addition, the functional maturation of invadopodia was illustrated by Transwell invasion assay indirectly. However, the lack of fluorescent gelatin-degradation assay is a limitation of the present study. In the present study, it was also found that p62 may be a negative regulator of invadopodia formation in human ovarian cancer SKOV3 cells. In a nutritionally deficient environment, autophagy directly induces the activation of ERK1/2 through p62, thus promoting cortactin phosphorylation and invadopodia formation. The authors aim to further investigate the mechanism through which autophagy acts on invadopodia in future research. 
p62 was initially isolated, and shown to be mainly interacting with atypical protein kinase $\mathrm{C}$. It functions as a selective autophagy receptor that recognizes and shuttles ubiquitinated proteins to the autophagosome for subsequent degradation in autophagy (10). Previous studies have revealed that p62 acts as a multidomain signaling hub through its ability to recruit, organize, and oligomerize important signaling molecules in cytosolic speckles, in order to control cell survival and apoptosis; moreover, the elimination of p62 by autophagy has been reported to suppress tumorigenesis $(13,15,17,30,31)$. The present study provides evidence of the role of p62 as an intermediary between autophagy and invasion. The fluctuation of p62 protein levels is dynamic during autophagy. It is described as the result of the interaction between two aspects: On the one hand, the p62 mRNA expression levels increased when cells were treated with EBSS, on the other hand, p62 degrades as autophagy progresses further $(16,17,22,23,30-32)$. In addition, ERK1/2 is a crucial kinase which participates in many cell activities, such as cell division, cell adhesion and apoptosis. . The variation in p-ERK1/2 levels may be attributed to their different location, combination and aggregation state in cells $(10,33-35)$. As a result, the regulatory mechanism is a complex phenomenon. Additionally, according to other previous studies, p62 expression exerts an inhibitory effect on ERK1/2 activity (13,23,36-38).

Little is known about the functional association between autophagy and invadopodia formation. Autophagy and invasion are different cell activities, and following EBSS treatment, their occurrences are not synchronous. Autophagy initially occurs, digesting part of the intracellular structure in order to ensure survival. Afterwards, invasion leads to the evasion of malignant cells from the adverse environment. This was a speculated selfprotective mechanism of tumor cells in the present study, which has been initially proved through the experiments. Autophagy was detected $2 \mathrm{~h}$ after EBSS treatment, and invadopodia formation was detected $8 \mathrm{~h}$ later. LC3 II marker level variance was used to evaluate autophagy, and a minimal increase in autophagy was detected at $24 \mathrm{~h}$, the authors aim to investigate this further in future studies, in order to elucidate whether there are other mechanisms involved. In addition, under various stimuli and at different time points, ERK1/2 participates in a number of cell activities. As a result, p62 expression did not decrease up to a certain level earlier than $8 \mathrm{~h}$, and ERK1/2 was not activated, a conclusion consistent with the invadopodia formation detection after the application of 8-h EBSS treatment.

Under conditions of nutritional deficiency, tumor cells enhance autophagy to provide nutrition for survival $(20,21,39)$, thus invading other tissues and organs to escape the nutrientdeficient environment. The present study demonstrated that autophagy may not only help cell survival, but may also increase tumor cell invasiveness under nutrient-deficient conditions. The interaction between autophagy and invasion may be a self-protective mechanism for tumor cells in a harmful environment. The underlying mechanisms of the autophagy and invasion interaction are being actively explored by the authors with the aim of gaining new insight regarding the prevention of tumor metastasis.

\section{Acknowledgements}

Not applicable.

\section{Funding}

The present study was supported by National Natural Science Foundation of China (grant nos. 81772794, 81672948, 81472419), Jilin Provincial Industrial Innovation Project (grant no. 2018C052-7) and the Fundamental Research Funds for the Central Universities, JLU.

\section{Availability of data and materials}

All data generated or analyzed during this study are included in this published article.

\section{Authors' contributions}

$\mathrm{ZZ}$ and JZ performed the experiments. YL, XY and HS collected and analyzed the data, MX and JS designed the study. All authors contributed to writing and reviewing the manuscript. ZZ and JZ confirm the authenticity of all the raw data. All authors have read and approved the final manuscript.

\section{Ethics approval and consent to participate}

Not applicable.

\section{Patient consent for publication}

Not applicable

\section{Competing interests}

The authors declare that they have no competing interests.

\section{References}

1. De Bock K, Mazzone M and Carmeliet P: Antiangiogenic therapy, hypoxia, and metastasis: Risky liaisons, or not? Nat Rev Clin Oncol 8: 393-404, 2011.

2. Fulda $S$ and Kögel D: Cell death by autophagy: Emerging molecular mechanisms and implications for cancer therapy. Oncogene 34: 5105-5113, 2015.

3. Hou J, Han Z, Zhao N and Wei L: Autophagy and tumour metastasis. Adv Exp Med Biol 1207: 315-338, 2020.

4. Perez-Montoyo H: Therapeutic potential of autophagy modulation in cholangiocarcinoma. Cells 9: 614, 2020.

5. Garcia J, Hurwitz HI, Sandler AB, Miles D, Coleman RL, Deurloo R and Chinot OL: Bevacizumab (Avastin ${ }^{\circledR}$ ) in cancer treatment: A review of 15 years of clinical experience and future outlook. Cancer Treat Rev 86: 102017, 2020.

6. Murphy DA and Courtneidge SA: The 'ins' and 'outs' of podosomes and invadopodia: characteristics, formation and function. Nat Rev Mol Cell Biol 12: 413-426, 2011.

7. Leong HS, Robertson AE, Stoletov K, Leith SJ, Chin CA, Chien AE, Hague MN, Ablack A, Carmine-Simmen K, McPherson VA, et al: Invadopodia are required for cancer cell extravasation and are a therapeutic target for metastasis. Cell Rep 8: 1558-1570, 2014.

8. Clark ES, Whigham AS, Yarbrough WG and Weaver AM. Cortactin is an essential regulator of matrix metalloproteinase secretion and extracellular matrix degradation in invadopodia. Cancer Res 67: 4227-4235, 2007.

9. Goertzen CG, Dragan M, Turley E, Babwah AV and Bhattacharya M: KISS1R signaling promotes invadopodia formation in human breast cancer cell via $\beta$-arrestin2/ERK. Cell Signal 28: 165-176, 2016.

10. Kelley LC, Hayes KE, Ammer AG, Martin KH and Weed SA: Revisiting the ERK/Src cortactin switch. Commun Integr Biol 4: 205-207, 2011 
11. Samuelson DR and Konkel ME: Serine phosphorylation of cortactin is required for maximal host cell invasion by Campylobacter jejuni. Cell Commun Signal 11: 82, 2013.

12. Navratil AM, Dozier MG, Whitesell JD, Clay CM and Roberson MS: Role of cortactin in dynamic actin remodeling events in gonadotrope cells. Endocrinology 155: 548-557, 2014.

13. Moscat J and Diaz-Meco MT: p62: a versatile multitasker takes on cancer. Trends Biochem Sci 37: 230-236, 2012.

14. Anand PK, Tait SW, Lamkanfi M, Amer AO, Nunez G, Pagès G, Pouysségur J, McGargill MA, Green DR and Kanneganti TD: TLR2 and RIP2 pathways mediate autophagy of Listeria monocytogenes via extracellular signal-regulated kinase (ERK) activation. J Biol Chem 286: 42981-42991, 2011.

15. Ciuffa R, Lamark T, Tarafder AK, Guesdon A, Rybina S, Hagen WJ, Johansen T and Sachse C: The selective autophagy receptor p62 forms a flexible filamentous helical scaffold. Cell Rep 11: 748-758, 2015.

16. Matsumoto G, Wada K, Okuno M, Kurosawa M and Nukina N: Serine 403 phosphorylation of p62/SQSTM1 regulates selective autophagic clearance of ubiquitinated proteins. Mol Cell 44: 279-289, 2011.

17. Ishimura R, Tanaka K and Komatsu M: Dissection of the role of p62/Sqstm 1 in activation of Nrf2 during xenophagy. FEBS Lett 588: 822-828, 2014

18. Mohamed NV, Plouffe V, Rémillard-Labrosse G, Planel E and Leclerc N: Starvation and inhibition of lysosomal function increased tau secretion by primary cortical neurons. Sci Rep 4: 5715,2014

19. Barutcu SA, Girnius N, Vernia S and Davis RJ: Role of the MAPK/cJun NH2-terminal kinase signaling pathway in starvation-induced autophagy. Autophagy 14: 1586-1595, 2018

20. Chang L, Chai X, Chen P, Cao J, Xie H and Zhu J: miR-181b-5p suppresses starvation-induced cardiomyocyte autophagy by targeting Hspa5. Int J Mol Med 43: 143-154, 2019.

21. Zhang Y, Ren S, Liu Y, Gao K, Liu Z and Zhang Z: Inhibition of Starvation-Triggered Endoplasmic Reticulum Stress, Autophagy, and Apoptosis in ARPE-19 Cells by Taurine through Modulating the Expression of Calpain-1 and Calpain-2. Int J Mol Sci 18: E2146, 2017.

22. Yan XY, Zhang Y, Zhang JJ, Zhang LC, Liu YN, Wu Y, Xue YN, Lu SY, Su J and Sun LK: p62/SQSTM1 as an oncotarget mediates cisplatin resistance through activating RIP1-NF- $\kappa$ B pathway in human ovarian cancer cells. Cancer Sci 108: 1405-1413, 2017.

23. Moscat J and Diaz-Meco MT: p62 at the crossroads of autophagy, apoptosis, and cancer. Cell 137: 1001-1004, 2009.

24. Roy R, Morad G, Jedinak A and Moses MA: Metalloproteinases and their roles in human cancer. Anat Rec (Hoboken) 303: 15571572,2020

25. Gonzalez-Avila G, Sommer B, García-Hernández AA and Ramos C: Matrix metalloproteinases' role in tumor microenvironment. Adv Exp Med Biol 1245: 97-131, 2020.
26. Cerofolini L, Fragai $M$ and Luchinat $C$ : Mechanism and inhibition of matrix metalloproteinases. Curr Med Chem 26: 2609-2633, 2019.

27. Eddy RJ, Weidmann MD, Sharma VP and Condeelis JS: Tumor cell invadopodia: Invasive protrusions that orchestrate metastasis. Trends Cell Biol 27: 595-607, 2017.

28. Yin M, Ma W and An L: Cortactin in cancer cell migration and invasion. Oncotarget 8: 88232-88243, 2017.

29. Martinez-Quiles N, Ho HY, Kirschner MW, Ramesh N and Geha RS: Erk/Src phosphorylation of cortactin acts as a switch on-switch off mechanism that controls its ability to activate N-WASP. Mol Cell Biol 24: 5269-5280, 2004.

30. Islam MA, Sooro MA and Zhang P: Autophagic regulation of p62 is critical for cancer therapy. Int J Mol Sci 19: E1405, 2018.

31. Moscat J, Karin M and Diaz-Meco MT: p62 in cancer: Signaling adaptor beyond autophagy. Cell 167: 606-609, 2016.

32. Sánchez-Martín P, Saito T and Komatsu M: p62/SQSTM1: 'Jack of all trades' in health and cancer. FEBS J 286: 8-23, 2019.

33. Cook SJ, Stuart K, Gilley R and Sale MJ: Control of cell death and mitochondrial fission by ERK1/2 MAP kinase signalling. FEBS J 284: 4177-4195, 2017.

34. Sun Y, Liu W-Z, Liu T, Feng X, Yang N and Zhou H-F: Signaling pathway of MAPK/ERK in cell proliferation, differentiation, migration, senescence and apoptosis. J Recept Signal Transduct Res 35: 600-604, 2015.

35. Roskoski R Jr: Targeting ERK1/2 protein-serine/threonine kinases in human cancers. Pharmacol Res 142: 151-168, 2019.

36. Gao H, Zhang Y, Dong L, Qu XY, Tao LN, Zhang YM, Zhai JH and Song YQ: Triptolide induces autophagy and apoptosis through ERK activation in human breast cancer MCF-7 cells. Exp Ther Med 15: 3413-3419, 2018.

37. Hou XO, Si JM, Ren HG, Chen D, Wang HF, Ying Z, Hu QS, Gao F and Wang GH: Parkin represses 6-hydroxydopamineinduced apoptosis via stabilizing scaffold protein p62 in PC12 cells. Acta Pharmacol Sin 36: 1300-1307, 2015.

38. Li C and Siragy HM: Autophagy upregulates (pro)renin receptor expression via reduction of P62/SQSTM1 and activation of ERK1/2 signaling pathway in podocytes. Am J Physiol Regul Integr Comp Physiol 313: R58-R64, 2017.

39. Gatica D and Klionsky DJ: Towards understanding mRNAbinding protein specificity: Lessons from post-transcriptional regulation of ATG mRNA during nitrogen starvation-induced autophagy. Curr Genet 65: 847-849, 2019.

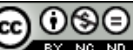

This work is licensed under a Creative Commons Attribution-NonCommercial-NoDerivatives 4.0 International (CC BY-NC-ND 4.0) License. 